\title{
MiR-449b-5p modifies glycolysis by lactate dehydrogenase A in gastric cancer
}

\author{
Yu Gao, Jingwang Ye, Dewen Tan, Haode Shen, Li Wang* \\ Department of General Surgery, Daping Hospital, Army Medical University, Chongqing, China
}

*For correspondence: Email: liwang516@yeah.net; Tel: +86-023-68757958

Sent for review: 20 August 2020

Revised accepted: 16 April 2021

\begin{abstract}
Purpose: To investigate the role of miR-449b-5p in gastric cancer (GC) metabolism. Methods: Human GC samples and their corresponding normal tissues were used in this study. Cell survival ability was evaluated using a commercial MTT kit. Glucose uptake, lactate production and ATP content were assessed using commercial kits. The expression levels of miRNAs were assessed by stem-loop reverse transcription-polymerase chain reaction (RT-PCR).

Results: MiR-449b-5p expression decreased in both GC tissues and cells. When the cells with low level of miR-449b-5p were transfected with miR-449b-5p mimics, glycolysis was inhibited $(p<0.05)$. Lactate dehydrogenase $A(L D H A)$ was predicted as a target gene of miR-449b-5p and verified using luciferase reporter assay. MiR-449b-5p expression was up-regulated in GC cells $(p<0.05)$. Furthermore, miR$449 b-5 p$ expression was related to long overall survival time in patients with GC.

Conclusion: The findings demonstrate that miR-449b-5p reverses the glycolytic state of GC cells by targeting LDHA expression, and thus, it can potentially be developed for the treatment of gastric cancer.
\end{abstract}

Keywords: Gastric cancer, Lactate dehydrogenase A, MiR-449b-5p

\begin{abstract}
This is an Open Access article that uses a fund-ing model which does not charge readers or their institutions for access and distributed under the terms of the Creative Commons Attribution License (http://creativecommons.org/licenses/by/4.0) and the Budapest Open Access Initiative (http://www.budapestopenaccessinitiative.org/read), which permit unrestricted use, distribution, and reproduction in any medium, provided the original work is properly credited.
\end{abstract}

Tropical Journal of Pharmaceutical Research is indexed by Science Citation Index (SciSearch), Scopus, International Pharmaceutical Abstract, Chemical Abstracts, Embase, Index Copernicus, EBSCO, African Index Medicus, JournalSeek, Journal Citation Reports/Science Edition, Directory of Open Access Journals (DOAJ), African Journal Online, Bioline International, Open-J-Gate and Pharmacy Abstracts

\section{INTRODUCTION}

Gastric cancer (GC) is a type of cancer of the digestion system. The major reasons for therapy failure in patients with GC include metastasis, chemotherapy drug resistance, tumor microenvironment and the glycolytic state [1]. GC patients have high recurrence and mortality. There is a high glycolytic state in GC tissues and many studies focus on cancer cell metabolism in order to find a new therapeutic method. However, there are still unknown pathological mechanism of GC for further investigation including, cancer cell glycolysis.
MicroRNAs (miRNAs) are a class of noncoding small RNA, which contain around 18 - 25 nucleotides. MiRNAs, important regulators of gene expression in various cells, suppress mRNA or protein levels of target genes by specifically binding and cleaving mRNAs or inhibiting their translation [2]. MiRNAs have been reported to be aberrantly expressed during pathogenesis and progression of GC [2]. Growing evidence shows that miRNAs involved in GC glycolysis include miR-21-5p, miR-520a-3p, miR-139-5p, miR-361-5p [3-6]. Some molecules involved in glycolysis such as 
hexokinase 2 (HK2), pyruvate kinase isozyme type M2 (PKM2), lactate dehydrogenase A (LDHA), glucose transporter 1 (GLUT1), etc, are regulated by miRNAs $[7,8]$, which suggests that miRNAs play a crucial role in cancer glycolysis.

LDHA is an important transcription factor related to tumor growth, resistance, metastasis and glycolysis [9], however, epigenetic therapy such as miRNAs for LDHA in GC is still unclear. In the present study, to assess the underlying mechanism of LDHA involved in GC glycolysis, biological informatics was used to predict miRNAs that might regulate LDHA expression. The new selected miR-449b-5p regulating LDHA will be further investigated to determine the molecular of action mechanism in GC.

\section{EXPERIMENTAL}

\section{GC tissue samples}

Human GC samples and their adjacent normal tissues were from the year of 2013 to 2014 . The tissue specimen collection and written consent from the persons concerned were approved by the Ethics Committee (Daping Hospital, Army Medical University, Chongqing, China). All participants were over 18 years of age and provided written informed consent for the publication of the data. The Human Research Ethic Committee at Fujian Medical University approved this study.

\section{Cell culture}

Cell lines of human GC cells and normal epithelial cell line were purchased from American Type Culture Collection (Mannasas, VA, USA). RGM-1 cells were cultured in Dulbecco's modified eagle medium (DMEM) with $10 \%$ Fetal Bovine Serum (FBS) and $1 \%$ penicillin/streptomycin. NCI-N87, CRL-5822, HGC-27, BGC-823, MGC-803, SGC-7901 and MKN45 cells were grown in Eagle's Minimum Essential Medium (Invitrogen), supplemented with $10 \%$ fetal bovine serum (HyClone, Logan, UT, USA) and $1 \%$ penicillin/streptomycin (Invitrogen).

\section{Luciferase assay}

The wild type LDHA 3'-untranslated region (UTR) containing miR-449b-5p targeting sequence and the mutated type was amplified and cloned into the luciferase reporter plasmid pGL4.1 vector (Promega, Madison, WI). All the constructs were verified by sequencing. The reporter plasmid and miR-449b-5p mimics were transfected into the cells and the cells were collected for luciferase assay using the kit from Promega (Madison, WI, USA) according to the manufacturer's protocol.

\section{Real time RT-PCR}

Cells were transfected with the miR-449-5p mimics or LDHA, and RNA was isolated using a Miniprep kit (Takara, Dalian, China). The expression levels of miRNAs were assessed by the stem-loop RT-PCR method using the Hairpin$\mathrm{it}^{\mathrm{TM}}$ miRNAs. qRT-PCR was performed using a TaKaRa SYBR RT-PCR kit (Takara, Dalian, China). The primer sequences of miR-449b-5p and U6 snRNA are listed in Table 1. Relative expression was calculated using the $\Delta \Delta \mathrm{Ct}$ method.

\section{MTT assay}

Cell survival ability was evaluated using a MTT kit (Solarbio, Beijing, China). MGC-803 and BGC-823 cells were transfected with miR-449-5p mimics or LDHA and then seeded in a 96-well plate $\left(2 \times 10^{4}\right.$ cells/well $)$. The cells were then used for MTT assay on 1, 3, 5 day. Absorbance at $490 \mathrm{~nm}$ was read using an automatic microplate reader.

\section{Western blot}

Total protein from cells or tissues were prepared in a lysis buffer; 50 ug protein samples were separated using SDS-PAGE, and then the protein bonds were transferred to a PVDF membrane (Millipore, USA). The membranes were incubated with primary LDHA (Abcam, USA) and GAPDH antibodies (Santa Cruz) overnight and then incubated with the secondary antibodies for $1 \mathrm{~h}$. The blots were visualized using the enhanced chemiluminescence $(E C L)$ reagent (Advansta, Menlo Park, CA, United States).

Table 1: The sequences of primers used for reverse transcription PCR

\begin{tabular}{lll}
\hline Variable & Primer & Sequence $\left(\mathbf{5}^{\prime} \rightarrow \mathbf{3}^{\prime}\right)$ \\
\hline \multirow{2}{*}{ miR-449b-5p } & Forward & ACACTCCAGCTGGGCCCAGATAATGGCAC \\
& Reverse & GTGCAGGGTCCGAGGT \\
\multirow{2}{*}{ 6 } & Forward & CTCGCTTCGGCAGCACA \\
& Reverse & AACGCTTCACGAATTTGCGT \\
\hline
\end{tabular}


Glucose uptake, lactate production and ATP content assays

Cells were transfected with the miR-449-5p mimics or LDHA and then seeded into 96-well plates for 24h. Glucose uptake was measured using the Glucose Assay Kit based on the protocols (BioVision, USA). The lactate from the supernatant was quantified using a colorimetric assay kit based on the instructions (Lactate Assay Kit, Biovision). Intracellular ATP content was measured using ATP assay kit (Beyotime, China).

\section{Statistical analysis}

Statistical analysis was performed using SPSS 15.0 (SPSS, Chicago, IL, USA) or Excel. Twosided Student's $t$ test was used to analyze differences between groups. Data are reported as mean $\pm \operatorname{SD}(n=4) . P<0.05$ was considered statistically significant.

\section{RESULTS}

MiR-449b-5p is predicted to regulate LDHA expression in GC

Ten miRNAs potentially binding to the 3'UTR of human LDHA were predicted using TargetScan 7.0. To identify the miRNAs that negatively regulate human LDHA in GC, individual miRNAs were transfected into MGC-803 cells and LDHA mRNA expression was tested by real time RTPCR. The data showed that LDHA mRNA was inhibited significantly in the cells with miR-449b$5 p$ (Figure 1 A). The expression of miR-449b-5p in GC was investigated. The results showed that the levels of miR-449b-5p were significantly lower in GC samples than the adjacent normal samples (Figure $1 \mathrm{~B}$ and $\mathrm{C}$ ). Further analysis from Kaplan-Meier analysis showed that the overall survival of GC patients with miR-449b-5p expression were significantly longer than the patients with low levels of miR-449b-5p expression (Figure $1 \mathrm{D}$ ). The results suggested that miR-449b-5p may a suppressor in the progression of human GC.

\section{MiR-449b-5p down-regulates expression in GC cells}

LDHA

To further determine whether LDHA expression is regulated by miR-449b-5p in GC cells, seven human GC cell lines were selected to detect miR-449b-5p expression. We found that in two GC cell lines including MGC-803 and BGC-823, miR-449b-5p expression was significantly lower than the RGM-1 normal cell line, however, in the other cell lines, miR-449b-5p expression decreased moderately (Figure $2 \mathrm{~A}$ ). The LDHA target sequences of miR-449-5p was shown (Figure 2 B). To confirm that miR-449b-5p regulates LDHA, MGC-803 and BGC-823 were transiently transfected with miR-449b-5p mimics and LDHA mRNA and protein were detected. The data from real time RT-PCR showed that endogenous LDHA mRNA levels decreased in MGC-803 and BGC-823 cells transfected with $m i R-449 b-5 p$ (Figure $2 \mathrm{C}$ ). The result from western blotting further confirmed that LDHA protein decreased in the MGC-803 and BGC-823 cells with miR-449b-5p (Figure 2 D). Results from luciferase activity assay showed that the promoter activity of LDHA (wide type) in SW480 cells was suppressed by miR-449b-5p, not changed for the mutated LDHA promoter (Figure $2 \mathrm{E})$. Combined with the data from Figure 1, it seems that LDHA is a target gene of miR-449b$5 p$ in GC cells.

\section{MiR-449b-5p inhibits GC cell proliferation and glycolysis by LDHA}

To assess the cellular survival of miR-449b-5p in GC cells, MGC- 803 and BGC-823 cells were transfected with miR-449b-5p and LDHA and MTT assay was used to assess cell survival of MGC-803 and BGC-823 cells with miR-449b-5p or LDHA transfection. The data from MTT assay showed that miR-449b-5p could decrease cell survival rates in MGC-803 and BGC-823 cells with LDHA overexpression (Figure $3 \mathrm{~A}$ and $\mathrm{B}$ ).

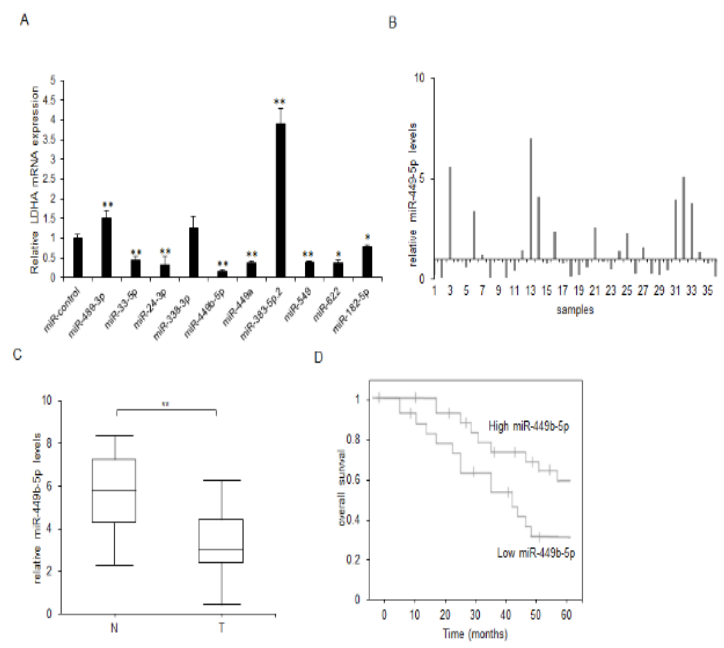

Figure 1: MiR-449b-5p regulates LDHA expression in GC cells. (A) The possible miRNAs binding to LDHA was shown. The predicted miRNAs were identified by targetscan and MicroCosm. (B) MiR-449b-5p expression in GC tissues. (C) The average expression of miR-449b-5p in GC tissues. (D) Kaplan-Meier analysis showed that the overall survival of GC patients with high and low miR-449b-5p expression 


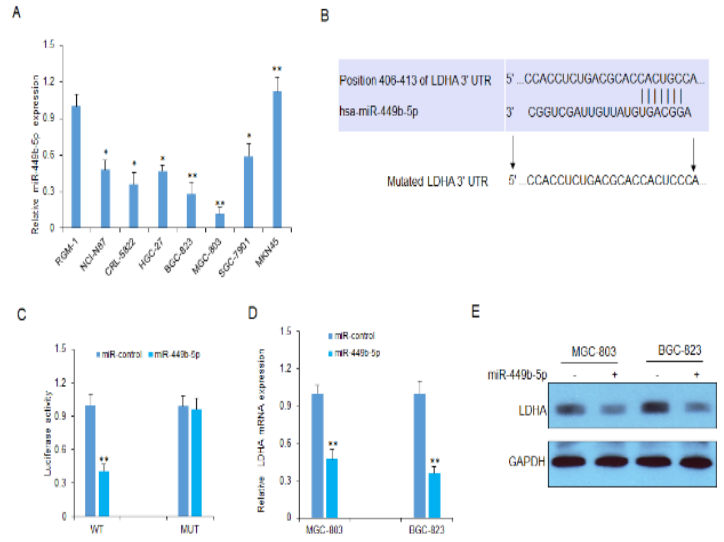

Figure 2: MiR-449b-5p down-regulates LDHA in GC cells. (A) Relative expression of miR-449b-5p in GC cell lines. (C) miR-449b-5p down-regulated LDHA mRNA in H2135 and H835 cells. H2135 and H835 cells were transfected with miR-449b-5p or miRNA control and then the total RNA was used for real time RT-PCR analysis. (D) (E) miR-449b-5p decreased luciferase activity of $L D H A$ promoter. miR-449b-5p down-regulated LDHA protein in $\mathrm{H} 2135$ and H835 cells. H2135 and H835 cells were transfected with miR-449b-5p or miRNA control and then the total protein was used for western blotting analysis

To determine whether miR-449b-5p is related to glycolysis in GC cells, metabolites from GC cells with miR-449b-5p or miRNA control combined LDHA were measured. Extracellular acidification rate (ECAR) index is the surrogate for glycolysis level. It was found that miR-449b-5p suppressed LDHA induced ECAR in MGC-803 and BGC-823 cells (Figure $3 \mathrm{C}$ and $\mathrm{D}$ ). Overexpression of LDHA in MGC-803 and BGC-823 cells increased cellular glucose uptake in miR-449b-5p overexpressed cells (Figure $3 \mathrm{E}$ and $\mathrm{F}$ ). It was shown that lactate production overexpression of LDHA in MGC-803 and BGC-823 cells increase cellular lactate production in miR-449b-5p overexpressed cells (Figure $3 \mathrm{G}$ and $\mathrm{H}$ ). Thus, compared with the control cells, miR-449b-5p cells consumed less glucose and extruded less lactate into the medium. The results show that LDHA is a direct target gene of miR-449b-5p, and its expression is critical and essential for miR-449b-5p-suppressed glycolysis.

\section{MiR-449b-5p is negatively related to LDHA in GC tissues}

To know the relationship between miR-449b-5p with LDHA mRNA in GC tissues. LDHA expression was measured in GC tissues and real time RT-PCR data showed that LDHA mRNA levels were higher in gastric cancer tissues than normal tissues (Figure $4 \mathrm{~A}$ ). The relationship between miR-449b-5p with LDHA mRNA was analyzed and it was shown that miR-449b-5p was negatively related to LDHA mRNA in GC tissues (Figure $4 \mathrm{~B}$ ). The data further confirmed that miR-449b-5p regulated LDHA expression in gastric cancer.
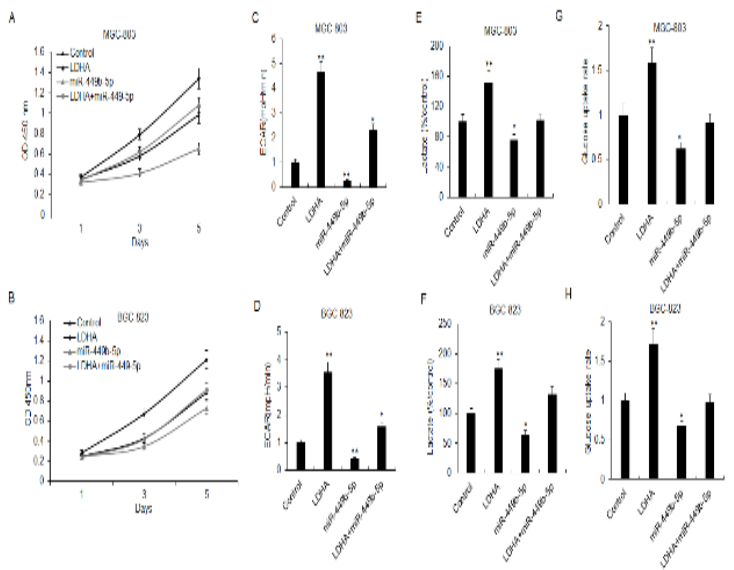

Figure 3: MiR-449b-5p inhibits GC cell proliferation and glycolysis. (A and B) GC cell proliferation was assay by MTT method. MiR-449b-5p or LDHA were introduced into MGC- 803 and BGC-823 cells by transfection and cell survival rates were assayed at 1 , 3 and 5 day. (C and D) ECAR measurement in MGC803 and BGC-823 cells. Cells were transfected with LDHA or miR-449b-5p for $24 \mathrm{~h}$ and then ATP was assayed. ( $E$ and $F$ ) Lactate measurement in MGC-803 and BGC-823 cells. Cells were transfected with LDHA or miR-449b-5p for $24 \mathrm{~h}$ and then lactate was assayed. (G-H) Glucose uptake in MGC-803 and BGC-823 cells. Cells were transfected with LDHA or miR-449b$5 \mathrm{p}$ for $24 \mathrm{~h}$ and then glucose uptake was assayed. Migration of MGC-803 and BGC-823 cells. (E and F) Invasion of MGC-803 and BGC-823 cells
A

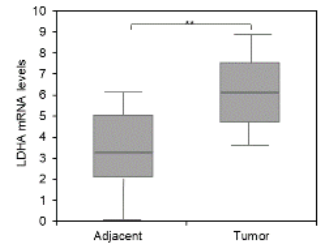

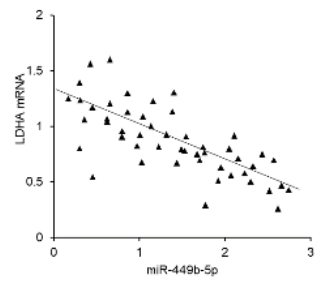

Figure 4: MiR-449b-5p was negatively related to LDHA in GC tissues. (A) LDHA expression in GC tissues. The RNA was isolated for LDHA expression evaluation by real time RT-PCR. (B) miR-449b-5p was negatively related LDHA mRNA in gastric cancer tissues $(r=-0.469)$

\section{DISCUSSION}

LDHA is an important molecule in cancer glycolysis, which converts pyruvate to lactate in the final step of glycolysis. LDHA is often higher in cancer, which involves in glucose metabolism, cell proliferation, metastasis, apoptosis, and 
immune reaction LDHA is regulated on posttranscriptional levels by miRNAs this study, we showed that LDHA was a target gene of miR$449 b-5 p$ which regulated glycolysis of GC cells. Our study demonstrated that miR-449b-5p significantly inhibited LDHA expression and provided a link between miR-449b-5p and LDHA expression in GC cells. The results, for the first time, revealed an important role of miR-449b-5p in glycolysis of GC cells.

MiR-449b-5p, has been found to be lost in several cancers including gastric cancer, breast cancer, nasopharyngeal carcinoma, lung adenocarcinoma and glioblastoma [10-14]. MiR$449 b-5 p$ inhibits gastric cancer cell proliferation, cell cycle, colony formation, and migration [10]. However, the biological role and precise mechanisms of miR-449b-5p in the progression of GC have not been reported until now. Therefore, in the present study, we investigated the exact roles of miR-449b-5p and its underlying molecular mechanisms in GC. In our study, we found that miR-449b-5p was significantly decreased, and forced expression of miR-449b$5 p$ inhibited cell proliferation, glucose consumption and lactate production in GC cells. These results suggest that miR-449b-5p functions as a tumor suppressor in this cancer. In addition, we found other miRNAs potentially regulate LDHA, but were not investigated in this study because some of them had been reported in GC.

Glycolysis, also called the Warburg effect, is a hallmark of malignant tumors. ATP production in glycolysis is less than in mitochondrial oxidative phosphorylation; however, energy in glycolysis is 100 times than it in oxidative phosphorylation [15]. In this study, we identified that miR-449b$5 p$ showed a suppressing role in glycolysis by targeting LDHA in GC cells. It was for the first time shown that miR-449b-5p decreased the lactate production, glucose consumption and extracellular acid rate.

\section{CONCLUSION}

The findings of this study demonstrate that the expression of miR-449b-5p is significantly decreased in GC, which leads to increase in the expression of LDHA, an important hypoxiainduced transcriptional factor. This, in turn, promotes cell proliferation and glycolysis in cancer. This finding provides a novel understanding of the molecular mechanism for LDHA-mediated glycolysis regulation. The novel roles of miR-449b-5p linked to glycolysis in GC suggests that miR-449b-5p might be a prognostic biomarker and potential target for future GC treatment.

\section{DECLARATIONS}

\section{Conflict of interest}

No conflict of interest is associated with this work.

\section{Contribution of authors}

We declare that this work was done by the authors named in this article and all liabilities pertaining to claims relating to the content of this article will be borne by the authors.

\section{Open Access}

This is an Open Access article that uses a funding model which does not charge readers or their institutions for access and distributed under the terms of the Creative Commons Attribution License (http://creativecommons.org/licenses/by/ 4.0) and the Budapest Open Access Initiative (http://www.budapestopenaccessinitiative.org/rea d), which permit unrestricted use, distribution, and reproduction in any medium, provided the original work is properly credited.

\section{REFERENCES}

1. Jiang $F$, Shen $X B$. miRNA and mRNA expression profiles in gastric cancer patients and the relationship with circRNA. Neoplasma 2019; 66: 879-886.

2. Alessandrini $L$, Manchi $M$, De Re V, Dolcetti $R$, Canzonieri V. Proposed Molecular and miRNA Classification of Gastric Cancer. Int J Mol Sci 2018; 19: 1683.

3. Liu Z, Yu M, Fei B, Fang X, Ma T, Wang D. miR-21-5p targets PDHA1 to regulate glycolysis and cancer progression in gastric cancer. Oncol Rep 2018; 40: 2955-2963.

4. Pan C, Liu Q, Wu X. HIF1a/miR-520a-3p/AKT1/mTOR Feedback Promotes the Proliferation And Glycolysis of Gastric Cancer Cells. Cancer Manag Res 2019; 11: 10145-10156.

5. Sun K, Hu P, Xu F. LINC00152/miR-139-5p regulates gastric cancer cell aerobic glycolysis by targeting PRKAA1. Biomed Pharmacother 2018; 97: 1296-1302.

6. Ma F, Zhang L, Ma L, Zhang Y, Zhang J, Guo B. MiR361-5p inhibits glycolytic metabolism, proliferation and invasion of breast cancer by targeting FGFR1 and MMP-1. J Exp Clin Cancer Res 2017; 36: 158.

7. Fang $R$, Xiao $T$, Fang $Z$, Sun $Y, L i F$, Gao $Y$, Feng $Y, L i$ L, Wang Y, Liu X, Chen H, Liu XY, Ji H. MicroRNA-143 (miR-143) regulates cancer glycolysis via targeting 
hexokinase 2 gene. J Biol Chem 2012; 287: 2322723235.

8. Sugiyama $T$, Taniguchi $K$, Matsuhashi $N$, Tajirika $T$, Futamura M, Takai T, Akao Y, Yoshida K. MiR-133b inhibits growth of human gastric cancer cells by silencing PKM-splicer PTBP1. Cancer Sci 2016; 107: 1767-1775.

9. Orang AV, Petersen J, McKinnon RA, Michael MZ. Micromanaging aerobic respiration and glycolysis in cancer cells. Mol Metab 2019; 23: 98-126.

10. Subramaniam S, Jeet V, Clements JA, Gunter JH, Batra $J$. Emergence of MicroRNAs as Key Players in Cancer Cell Metabolism. Clin Chem 2019; 65: 1090-1101.

11. Qu W, Ding SM, Cao G, Wang SJ, Zheng XH, Li GH. miR-132 mediates a metabolic shift in prostate cancer cells by targeting Glut1. FEBS Open Bio 2016; 6: 735741.

12. $Y u L$, Chen $X$, Wang $L$, Chen $S$. The sweet trap in tumors: aerobic glycolysis and potential targets for therapy. Oncotarget 2016; 7: 38908-38926.

13. Zhao Y, Lu G, Ke X, Lu X, Wang X, Li H, Ren M, He S. miR-449b-5p acts as a tumor suppressor gene in gastric cancer. Tumour Biol 2016; 37: 8691-8698.

14. Jiang J, Yang $X$, He X, Ma W, Wang J, Zhou Q, Li M, Yu S. MicroRNA-449b-5p suppresses the growth and invasion of breast cancer cells via inhibiting CREPT- mediated Wnt/ $\beta$-catenin signaling. Chem Biol Interact 2019; 302: 74-82.

15. Yin W, Shi L, Mao Y. MicroRNA-449b-5p suppresses cell proliferation, migration and invasion by targeting TPD52 in nasopharyngeal carcinoma. J Biochem 2019; 166 : 433-440.

16. Yue $N$, Ye M, Zhang R, Guo Y. MiR-449b-5p targets IncRNA PSMG3-AS1 to suppress cancer cell proliferation in lung adenocarcinoma. BMC Pulm Med 2020; 20: 152.

17. Hou WZ, Chen XL, Qin LS, Xu ZJ, Liao GM, Chen D, Hu LJ, Mao ZM, -S Huang J, Yuan Q, Liao C, Yao QM. MiR-449b-5p inhibits human glioblastoma cell proliferation by inactivating WNT2B/Wnt/ $\beta$-catenin signaling pathway. Eur Rev Med Pharmacol Sci 2020; 24: 5549-5557.

18. Liu Y, Zhang Z, Wang J, Chen C, Tang X, Zhu J, Liu J. Metabolic reprogramming results in abnormal glycolysis in gastric cancer: a review. Onco Targets Ther 2019; 12 : 1195-1204.

19. Tam C, Wong JH, Tsui SKW, Zuo T, Chan TF, Ng TB. LncRNAs with miRNAs in regulation of gastric, liver, and colorectal cancers: updates in recent years. Appl Microbiol Biotechnol 2019; 103: 4649-4677.

20. Yuan LW, Yamashita $H$, Seto $Y$. Glucose metabolism in gastric cancer: The cutting-edge. World J Gastroenterol 2016; 22: 2046-2059. 